\title{
Glassy carbon electrode modified by molybdenum to Detection Hydrochlorothiazide
}

\author{
Ahmad Khalaf Alkhawaldeh ${ }^{1}, *$, Rawia Alkhawaldeh ${ }^{2}$ \\ ${ }^{1}$ Ahmad University of Jordan, Department of Chemistry, Amman - 11942, Jordan. E-mail: \\ Ahm9140096@fgs.ju.edu.jo, Ahmad.alkawalda@yahoo.com \\ ${ }^{2}$ Rawia Department of Mechatronics Engineering, Al-Balq'a Applied University, Amman 11134- Jordan, E-mail: \\ RKH88RKH88@gmail.com
}

Corresponding Author: ahmad.alkawalda@yahoo.com

\begin{abstract}
Molybdenum oxides is well-known electro-catalysts in fuel cells systems, they are usually used as anodic materials for the oxidation of low molecular weight alcohols. The utilization of Mo as catalysts in the pharmaceutical analysis is not common yet. In this study, bare glassy carbon electrodes were modified by the oxides by means of electrochemical deposition and the modified electrodes were used as catalysts for the electrochemical oxidation of hydrochlorothiazide (HCT). Well-resolved anodic peaks were reported for the analyzed pharmaceuticals when the Mo/GCE was utilized for the analysis of HCT. Analytical performance of the modified electrodes was evaluated based on the following statistical parameters; linearity ranges, correlation coefficients, limits of detection and quantitation, and recovery values. The prepared electrodes were used for the determination of the active ingredients in their pharmaceutical formulations and the reported activity was correlated to influence of the utilized $\mathrm{pH}$ on both structures of the used electrodes and the detected analytes.

Keywords: Hydrochlorothiazide; Differential pulse voltammograms; Cyclic Voltammetry; Molybdenum; Modified electrode
\end{abstract}

\section{Introduction}

In the 16th century, the role of diuretics began when they were solely introduced to remove fluid accumulation in the body for the treatment of edema. Later, in 1957, Sharp and Dohme (now Merck) modernized the concept of diuretic therapy by the introduction of thiazides, as anti-hypertensive agents, which proved to lower blood pressure as a secondary consequence (Altwaiq, et al., 2015). A prototypic member, Hydrochlorothiazide (HCT), was first sold commercially in early 1959 and still remains to be the most widely prescribed drug of thiazide class for the treatment of hypertension and edema. This is because HCT has a half-life of 8-12 hours, which permits effective oncedaily doses in comparison with all other thiazide diuretics (Alshamaileh et al., 2016).

HCT is only administered orally in three doses: $12.5,25$, and $50 \mathrm{mg}$ tablets. Following oral administration, the drug is well absorbed (65-75\%) and 55\%-77\% of the administered dose appears in the urine. In addition, more than $95 \%$ of the absorbed dose is excreted in urine as an unchanged drug (i.e. not metabolized) within 24 hours (Altweiq and Alkhawaldeh, 2019).

HCT is chemically designated as 6-Chloro-3,4-dihydro-2H-1,2,4-benzothiadiazine-7-sulfonamide 1,1dioxide with five main functional groups: an aromatic ring, halide group, secondary amino group, cyclic and acyclic sulfonamide groups. Industrially, the drug is synthesized by either the reaction of para-formaldehyde with 5-chloro-2,4disulfamoylaniline in nonaqueous media or the reaction of formaldehyde with 6-chloro-7-sulfamoyl-2H-1,2,4benzothiadiazine-1,1-dioxide in an aqueous alkaline solution of ammonia (Hourani and Alkawaldeh, 2016).

The utilization of manganese and molybdenum oxides as oxidizing agents in pharmaceutical electrocatalysis is not common. These oxides have electron deficient metallic centers of high oxidation states; therefore, carbon-based electrodes modified with these oxides have the potential to oxidize a wide range of organic compounds such as alcohols and alkanes more efficiently than their bare counterparts. Molybdenum modified electrodes are usually used for promotion of methanol and ethanol oxidation in fuel cells systems where the alcohol adsorption and the following oxidation is enhanced in the presence of molybdenum. Molybdenum is utilized as co-catalyst besides the key 
fuel cells catalysts such as platinum, platinum-based alloys, and complexes with platinum as the metallic center (Alkhawaldeh et al, 2020).

In this work, glassy carbon electrodes were modified with molybdenum and manganese oxides by means of electrochemical deposition and the modified electrodes were used for HCT determination in locally provided pharmaceutical dosage forms. Details of the modification and the assay will be demonstrated within the context of the following sections.

\section{Experimental}

The voltammetric methods are based on measuring the current produced while changing the potential as a function of time, and its data comes in form of voltagramm, which plots the current produced versus the potential of the working electrode. The modern three electrode system as shown in Figure 1, which consists of working, reference and counter electrodes. The potential is applied between the working and the reference electrodes several methods were used in electrochemical deposition of the surface as linear scan voltammetry, Cyclic Voltammetry, and Differential pulse voltammetry.

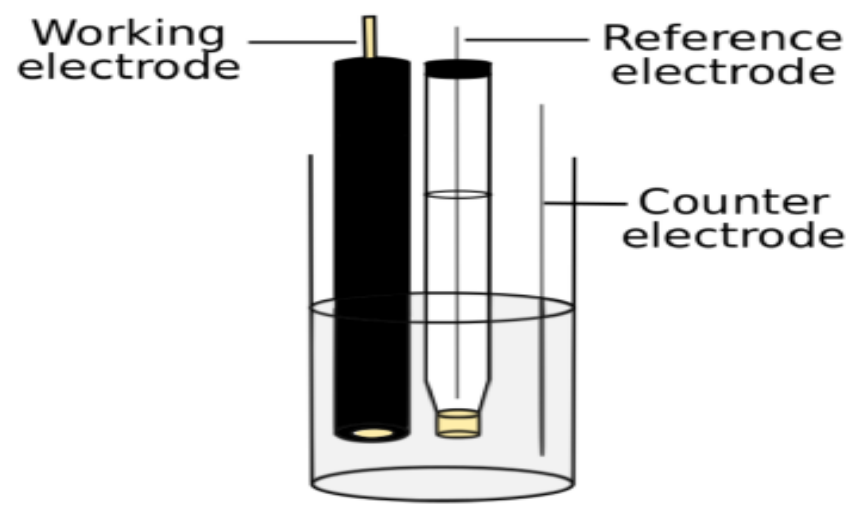

Figure 1. Modern three electrodes system

The chemicals used in this work are $\mathrm{H}_{3} \mathrm{BO}_{3}$ (Boric acid) from Riedel-de Haën, $\mathrm{H}_{3} \mathrm{PO}_{4}$ (Phosphoric Acid) from VWR, $\mathrm{CH}_{3} \mathrm{COOH}$ (Acetic Acid), and $\mathrm{NaOH}$ (Sodium Hydroxide) from VWR, all solutions were prepared using ultrapure water, solutions were prepared by dissolving in britton Robinson buffer $\mathrm{pH}=7$ to known concentrations. The Septrin and Balkatrin drugs were purchased from Janssen chemical.

Sigma-Aldrich Chemie GmbH, Darmstadt, Germany, provided hydrochlorothiazide. VWR chemicals provided all sodium hydroxide, potassium chloride, boric acid, and phosphoric acid, NY, U.S.A. Glacial acetic acid, sodium molybdate, sodium sulfate, and potassium permanganate were purchased from S D Fine-Chem Ltd., Mumbai, India. The commercial products (Brand A, Brand B, Brand C, and Brand D) were purchased from the local market.

\section{Methodology}

Modification of the electrode surface was carried out by $\mathrm{CV}$ of the electrode into a solution that contains the cation of the element at open circuit conditions. The concentration of standard solutions $\left(1 \times 10^{-3} \mathrm{M}\right)$ was prepared to study the voltammetric response of those compounds. Cyclic voltammograms where scanned in a relatively limited potential window from -1.0 to $0.2 \mathrm{~V}$. Open-circuit adsorption by cyclic voltammetry of Cadmium, Manganese and Molybdenum on glassy carbon was done. No deterioration was observed in the adatoms (Molybdenum) monolayer during experimentation for many hours, but because of the simplicity of preparation of the adatoms-coated electrode, re-coating the surface was followed on daily basis.

A clean glassy carbon surface electrode (Molybdenum) were reproduced and potentiosatated. The potential was held constant for 3-5 minutes followed by rinsing the electrode 3 times then the potential was scanned between -0.2 and $1.2 \mathrm{~V}$. Cyclization was repeated many times until the potential window between -0.2 and $1.2 \mathrm{~V}$ showed the absence of any faradic current. Cadmium was found to adsorb irreversibly on carbon electrodes surface at open circuit. The 
experiments were conducted from $10^{-3} \mathrm{M}$ Cadmium in solution. The exposure time of the electrode to the abovementioned solution was 2 minutes. The prepared carbon electrode was analyzed by cyclic voltammetry.

Stock solution consists of a mixture of $0.04 \mathrm{M} \mathrm{H}_{3} \mathrm{BO}_{3}, 0.04 \mathrm{M} \mathrm{CH}_{3} \mathrm{COOH}$ and $0.04 \mathrm{M} \mathrm{H}_{3} \mathrm{PO}_{4}$. Initially a stock solution was prepared from which $10^{-3} \mathrm{M}$, which has been titrated to the desired $\mathrm{pH}$ with $0.2 \mathrm{M} \mathrm{NaOH}$.

\section{Results and Discussion}

Both currents, faradaic and capacitive will decrease with time so the sampling should be taken in the late pulse life time until most of the capacitive current decay, because capacitive current decays faster as shown in Figure 2, so the net current will equal almost the faradaic current. Regarding the scan rate, if the reaction is fast reaction, then the scan rate must be high the reaction is not fast so the scan rate used is not high.

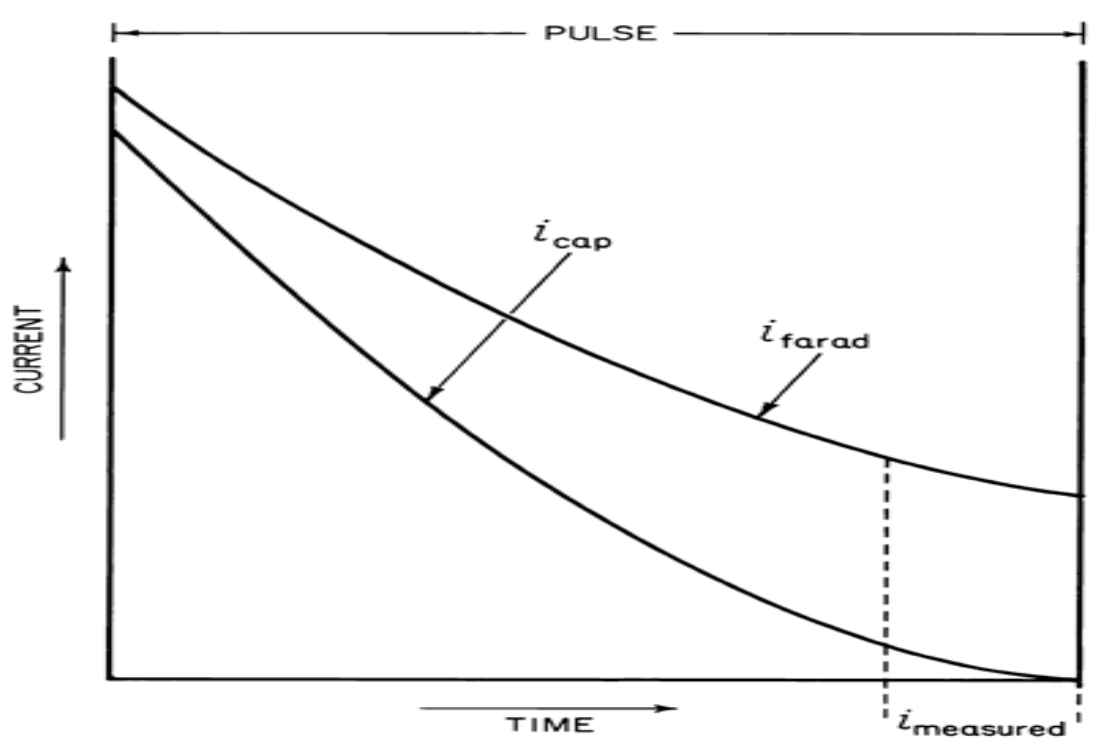

Figure 2. Faradaic and capacitive currents decay

The prepared molybdenum coated glassy carbon electrode by cyclic voltammetry was analyzed by SEM. Figures 3 and 4 show the SEM micrographs of glassy carbon electrode pure without any modified and glassy carbon electrode modified by molybdenum, respectively. Investigation of the SEM micrographs shows that the particle size, shape and distribution on the surface are dependent on cyclic voltammetry. Moreover, the SEM micrographs show enhanced deposition along the grain boundaries and a variation in particle size.

The results of this work powerfully supports that particle growth is that the prevailing mechanism of metal deposition at high concentrations and long deposition times. The initial stages of deposition involve nucleation, which happens by selection at high-energy surface sites. These high-energy surface sites embody surface defects, voids, adatoms, or grain boundaries. At low particle concentration, nucleation prevails due to the supply of high-energy surface sites. 


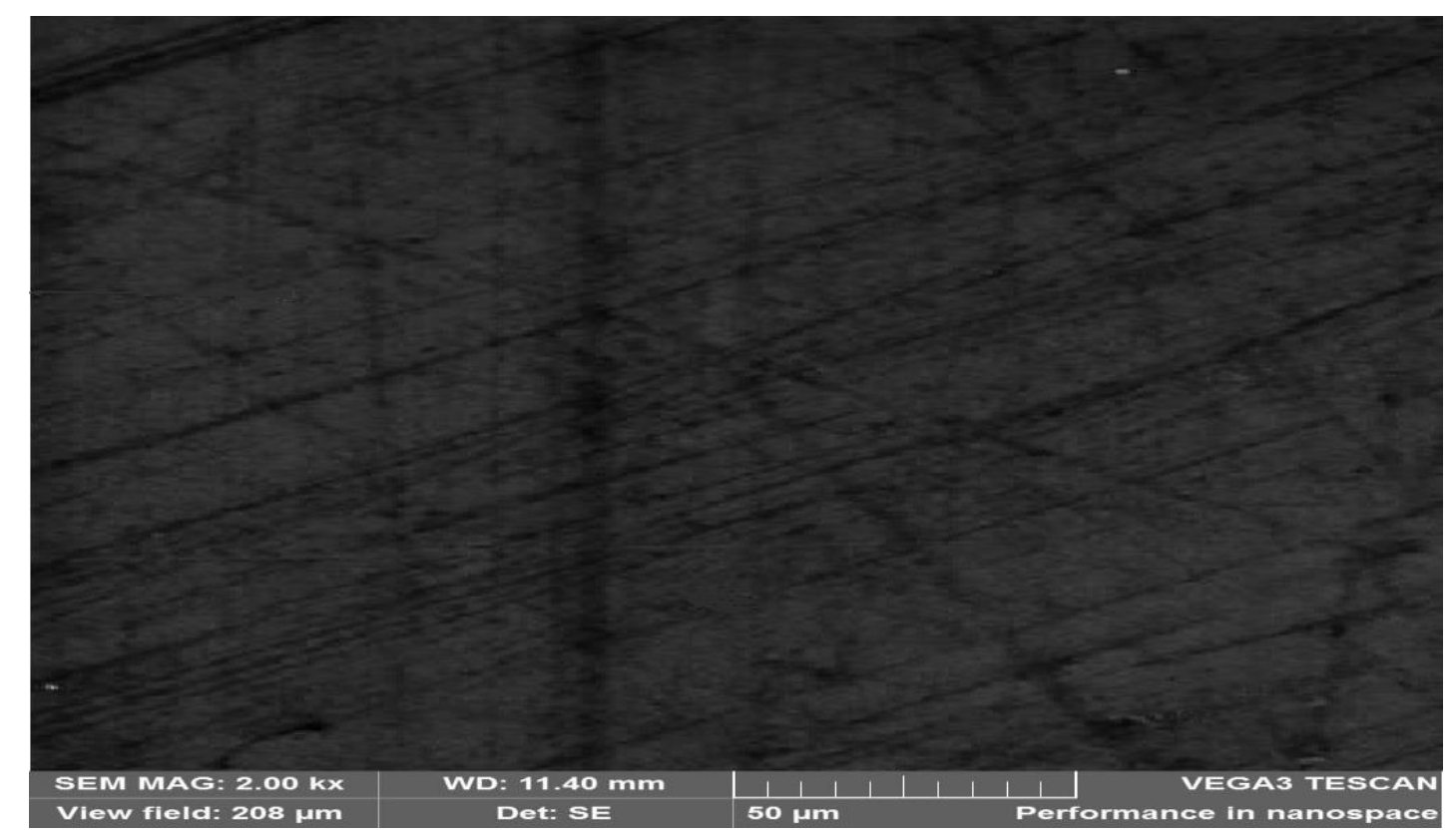

Figure 3. SEM images of bare glassy carbon electrode.

On the opposite hand, at high concentration of depositing ions, high surface energy sites square measure comparatively restricted so creating particle growth mechanism current. On identical basis, the result of deposition time is often explained. At short deposition times, nucleation mechanism prevails due to the supply of highenergy surface sites, whereas particle growth prevails once most of those sites square measure consumed at long deposition times. This might essentially enhance the formation of 3-dimentional structures, at long deposition times.

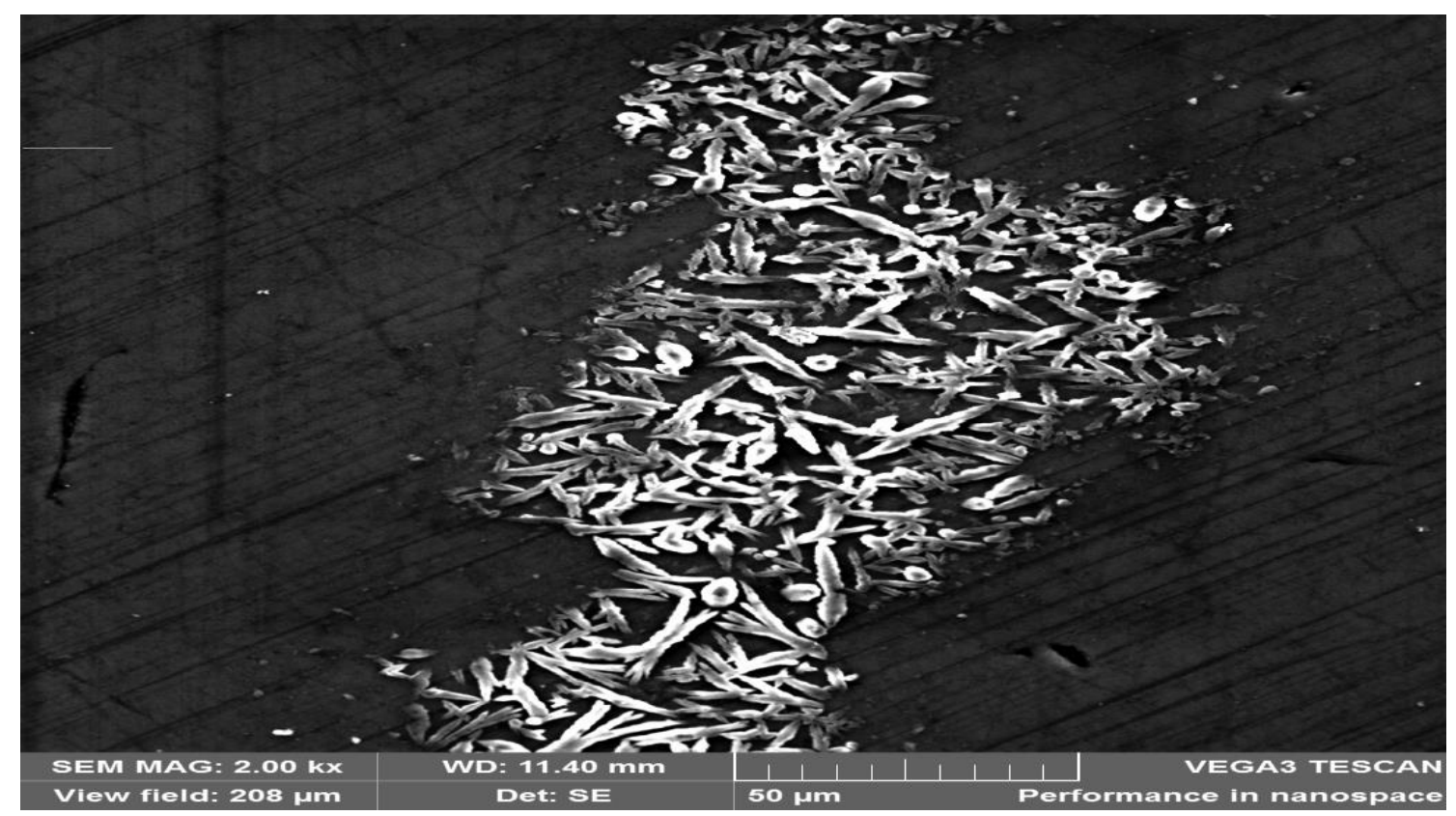

Figure 4. SEM images of glassy carbon electrode modified by molybdenum.

Table 1 shows the analytical performance of the differential pulse voltammograms of the hydrochlorothiazide in the cell, by using molybdenum coated glassy carbon electrode, twelve differential pulse voltammograms of the same 
glassy carbon electrode modified by Molybdenum recorded at a concentration between $9.80 \times 10^{-4} \mathrm{M}$ to $9.02 \times 10^{-6} \mathrm{M}$ of hydrochlorothiazide.

Table 1. The concentration of hydrochlorothiazide at $\mathrm{Mo}_{\mathrm{ad}}$-glassy carbon electrode.

\begin{tabular}{|c|c|c|}
\hline $\begin{array}{l}\text { Volume of } \\
\text { hydrochlorothiazide }(\mathrm{ml})\end{array}$ & $\begin{array}{c}\text { Concentration of hydrochlorothiazide } \\
\text { on bara glassy carbon electrode }\end{array}$ & $\begin{array}{l}\text { Concentration of hydrochlorothiazide on } \\
\qquad \mathrm{Mo}_{\mathrm{ad}-\text { glassy carbon electrode }}\end{array}$ \\
\hline 0.5 & $9.80 \times 10^{-6}$ & $1.94366 \times 10^{-6}$ \\
\hline 1 & $1.53 \times 10^{-5}$ & $2.4472 \times 10^{-6}$ \\
\hline 1.5 & $2.83 \times 10^{-5}$ & $2.83966 \times 10^{-6}$ \\
\hline 2 & $3.70 \times 10^{-5}$ & $3.16528 \times 10^{-6}$ \\
\hline 2.5 & $4.55 \times 10^{-5}$ & $3.43994 \times 10^{-6}$ \\
\hline 3 & $5.36 \times 10^{-5}$ & $3.67737 \times 10^{-6}$ \\
\hline 3.5 & $6.14 \times 10^{-5}$ & $3.85193 \times 10^{-6}$ \\
\hline 4 & $6.90 \times 10^{-5}$ & $4.00 \times 10^{-6}$ \\
\hline 4.5 & $7.63 \times 10^{-5}$ & $4.21967 \times 10^{-6}$ \\
\hline 5 & $8.33 \times 10^{-5}$ & $4.38019 \times 10^{-6}$ \\
\hline 5.5 & $9.02 \times 10^{-5}$ & $4.52389 \times 10^{-6}$ \\
\hline
\end{tabular}

The voltammograms show a change in the peak absorption of the electrode with increasing the concentration of hydrochlorothiazide. Table 1 , it is evident that the peak current increases with increasing concentration of hydrochlorothiazide in the solution.

It also explains the change in the voltammograms with results list current of anodic peaks; this indicates that the glassy carbon electrode modified by Molybdenum is sensitive to hydrochlorothiazide. This indicates the response of the increases as the concentration increase. A linear response of hydrochlorothiazide current appears to be within the range of 0.5 to $5.5 \mathrm{ml}$ as shown in the table. The relationships between the volume and the concentrations of hydrochlorothiazide in the bulk are linear with a regression coefficient of 0.995 and 0.960 for hydrochlorothiazide.

The use of glassy carbon electrodes modified by Molybdenum can detect as $2 \times 10^{-6} \mathrm{M}$ for hydrochlorothiazide the solution. At lower concentrations, such as $2 \times 10^{-5} \mathrm{M}$ a small noticeable peaks change on the voltammograms and smaller peaks were observed at $0.88 \mathrm{~V}$ and $1.1 \mathrm{~V}$ on the voltammograms. This means that Molybdenum coated glassy carbon electrode is still sensitive at this concentration, going down to $1 \times 10^{-7} \mathrm{M}$ concentration where no noticeable change on the voltammograms is observed. This indicates the absence of response of the electrode at this concentration. 
The oxidation of HCT at the manganese-modified electrode is presented in Figure 5. The oxidation current at the modified surface is three times higher than that reported at the bare electrode. The conductive nature of the added modifier is one of the two factors behind the reported activity. The other factor is the role of the manganese oxide as both oxidizing agent and catalyst. Voltammograms of the manganese-modified electrode in HCT buffered solutions are presented. The highest catalytic activity was reported at $\mathrm{pH} 3.00$; the highest oxidation peak is shown at $1183 \mathrm{mV}$ and the height of the peak decreases as the $\mathrm{pH}$ increases. At $\mathrm{pH} 12.2$, the peak has disappeared entirely.

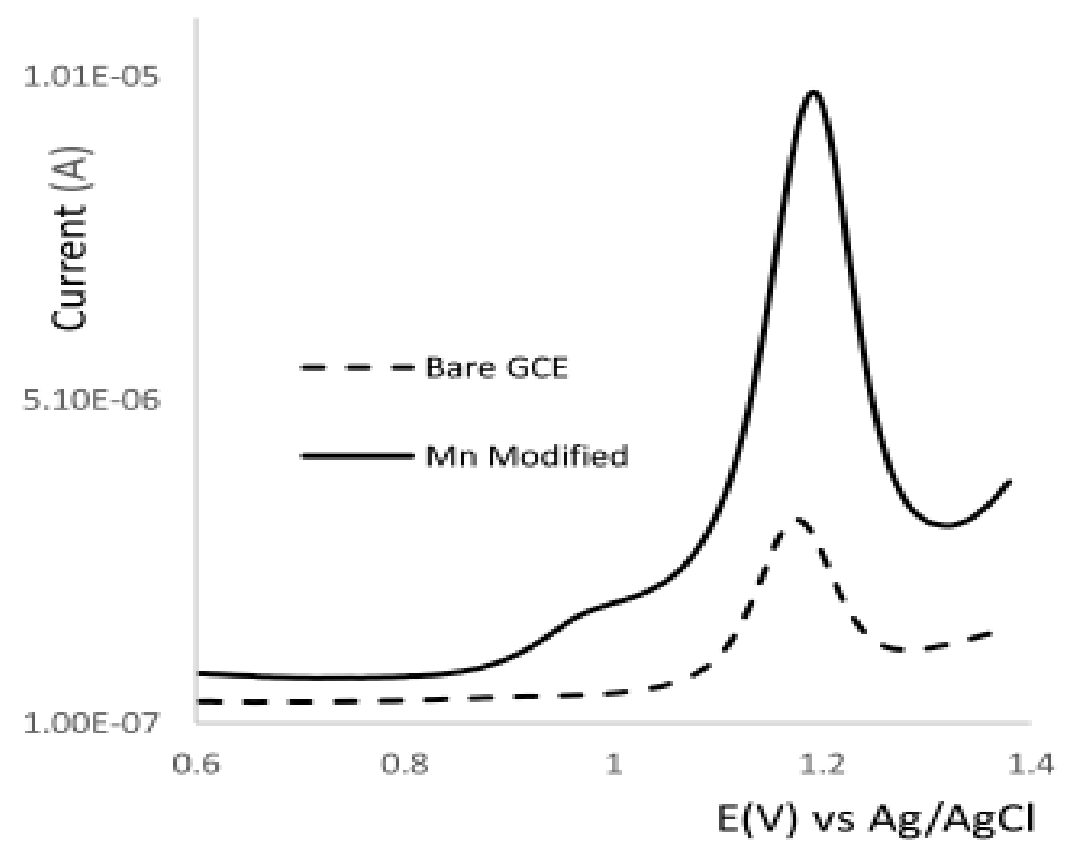

Figure 5. Differential pulse voltammograms of the bare and the manganese-modified glassy carbon electrode in pH: 3 in buffer solution containing $2.0 \mathrm{mM} \mathrm{HCT}$

Table 2. Comparison of activity of Mn/GCE with other modified electrodes for HCT determination

\begin{tabular}{|c|c|c|c|}
\hline Electrode & Technique & Linear Range $(\mu \mathrm{M})$ & LOD $(\mu \mathrm{M})$ \\
\hline $\mathrm{Mn} / \mathrm{GCE}$ & DPV & $3.00 \times 10^{2}-1.00 \times 10^{3}$ & 5.86 \\
& & & \\
\hline
\end{tabular}

\section{Conclusion}

HCT is a diuretic. Manganese and molybdenum oxides have electron deficient metallic centers; therefore, they have the potential to oxidize the pharmaceuticals. In addition, the metal oxides enhance the electron transfer rate across the electrode-electrolyte interface, since they are more conductive than bare glassy carbon electrodes. The proposed catalytic activity was correlated to the utilized $\mathrm{pH}$ that tunes structure of the target analyze as well as surface composition of the modified electrodes. Performance of the utilized electrodes could be improved by enhancement of their conductivity and variation of thickness of the acting catalysts, which may be carried out by manipulation of the electro-deposition conditions, such as nature of the electrochemical deposition signal, concentrations of the added modifiers, and $\mathrm{pH}$ of the deposition solutions. 


\section{References}

Alkhawaldeh, A. K. (2020). Analytics of Antimony in Natural Water of Nanoparticle Platinum Electrode by Application Square Wave Voltammetry, International Journal of Multidisciplinary Sciences and Advanced Technology, 1(4): 96-103.

Alkhawaldeh, A. K. (2020). Electrical Conductivity of Natural Volcanic Tuff Mix by Cyclic Voltammetry Method, International Journal of Multidisciplinary Sciences and Advanced Technology, 1(5): 37-44.

Alkhawaldeh, A. K. (2020). Platinum Nanoparticle in Tantalum Electrode for the Electrochemical Analysis of Heavy Metal Ions, International Journal of Intelligent Computing and Technology, 4(1): 25-35.

Alkhawaldeh, A. K. (2020). Platinum Nanoparticles for the Electrochemical Study of Heavy Metal ions Formed by the Sputtering Deposition of the ion beam Electrode. International Journal of Engineering and Artificial Intelligence. 1(3): $1-8$.

Alkhawaldeh, A. K. Alkhawaldeh, R. (2020). Highly Sensitive copper Heavy Metal Analysis on Nanoparticle Platinum and palladium electrode, International Journal of Engineering and Artificial Intelligence. 1(2): 33-39.

Alkhawaldeh, A. K., M.Krishan, M., Altwaiq, A., Dabaibeh, R. N. (2020). Preparation of Nanostructured/ Microplatinum Surfaces by Application of a Square Wave Potential Regime for Methanol Oxidation. Eurasian Journal of Analytical Chemistry, 15(1), emEJAC-00362.

Almatarneh, M. H., Elayan, I. A., Al-Sulaibi, M., Khawaldeh, A., Saber, S. O. W., Al-Qaralleh, M., and Altarawneh, M. (2019). Unimolecular Decomposition Reactions of Propylamine and Protonated Propylamine. ACS Omega, 4(2), 33063313. doi: 10.1021/acsomega.8b02792.

Alshamaileh, E., Al-Sulaibi, M., Al-Khawaldeh, A., Almatarneh, M., El-Sabawi, D. and Al-Rawajfeh, A. (2016), "Current status of nanotechnology in Jordan", World Journal of Science, Technology and Sustainable Development, Vol. 13 No. 2, pp. 66-81. https://doi.org/10.1108/WJSTSD-01-2016-0001.

Altweiq, A. and Alkhawaldeh, A (2019). The Determination of Some Heavy Metals in Different Selected Diets. Eurasian Journal of Analytical Chemistry, 14(4), emEJAC-00326.

Al-Zboon, K. K., Al-Smadi, B. M., \& Al-Khawaldh, S. (2016). Natural Volcanic Tuff-Based Geopolymer for Zn Removal: Adsorption Isotherm, Kinetic, and Thermodynamic Study. Water, Air, \& Soil Pollution, 227(7). doi:10.1007/s11270-016-2937-5.

Hourani, M. K. and Alkawaldeh A. (2016). Synergistic Effects of Bismuth Adatoms on Electrocatalytic Properties of Electrodeposited Nanostructured Platinum Electrodes. International Journal of Electrochemical Science, 3555-3566. doi: $10.20964 / 110434$.

Mathiyarasu, J. Pathak, S. S.; Yegnaraman, V. (2016), Review on corrosion prevention of copper using ultrathin organic monolayers. Corrosion Reviews, 24, 307-321.

Pettinger, B. Schoppel, R. H and Gerishcer, H. (2018). Tunneling effects in the spectral sensitization on highly doped zinc oxide electrodes, Ber.Bunsenges .physik.Chem, 77, 960-966.

Sgobbi F. Razzino.C. A., Machado. S.A.S, (2016). A disposable electrochemical sensor for simultaneous detection of sulfamethoxazole and trimethoprim antibiotics in urine based on multiwalled nanotubes decorated with Prussian blue nanocubes modified screen-printed electrode. Elsevier Electrochemica acta, 191: 1010-1017. 